\title{
Fermented Foods in Medicine: An Evaluation of Their Uses and Effects on the Human Gut Microbiota ${ }^{1}$
}

\author{
Elizabeth Storm ${ }^{2}$
}

\begin{abstract}
This paper reviews several health-related claims about fermented foods as they relate to the human gut microbiome. Beyond regular health benefits, these claims include the ability of fermented foods and their products to reduce the risk of disease. Several immune-related diseases, infectious diseases, and metabolic disorders are linked to an unhealthy gut microbiome, where the effects of fermented foods allow for the restoration of a healthy microbiome to induce a state of overall well-being. This review identifies and clarifies the validity of the claims made in favor of fermented foods through investigating the uses of fermentation in medicine and their impact on the human gut microbiome. The future of fermentation products in medicine holds great promise for novel treatments. Further research may provide invaluable knowledge on the connection between the gut microbiome and overall health.
\end{abstract}

Keywords: Fermentation, fermented products, types of fermentation, gut microbiome, pharmaceuticals, food products, human health, humans

\section{Introduction}

Fermentation is the (generally) anaerobic conversion of organic compounds to more oxidized chemicals (e.g., acetic acid, ethanol, lactic acid, and many others) by bacteria or fungi, with the generation of a relatively small amount of energy. Besides its widespread uses in food production (e.g., alcohol used in beverages; cheese, kefir, kimchi) and agriculture (e.g., pesticides, such as the fermentation used in the production of Bacillus thurigiensis endotoxins for use as a biopesticide, Capalbo 1995), fermentation has a long history of uses in medicine (e.g., analgesics, or pain-relieving drugs, and disinfectants) (Rahman 2013, International Service for the Acquisition of Agri-biotech Applications 2006).

Public interest in fermentation within the health community is growing (Rahman 2013). In his book, The Art of Fermentation, Sandor Katz provides extensive knowledge and background on the processes of fermentation around the world, characterizing not only the traditional foods and methods but also introducing new and innovative ideas for the future of fermented foods (Katz 2012). This book exemplifies the widespread interests and historical uses of fermentation across the globe. With the ever-emerging technologies of molecular biology and extensive research efforts, fermentation fulfills a broader range of medical uses, such as improved digestive health and reduction of pathogenic

\footnotetext{
${ }^{1}$ Submitted on March 11, 2020. Accepted on May 30, 2020. Last revisions received on August 17, 2020.

${ }^{2}$ Elkridge, Maryland, USA. E-mail: eastorm13@gmail.com .
}

DOI: $10.9784 /$ LEB8(2)Storm.01

Electronically available on August 31, 2020. Mailed on August 31, 2020. 
bacteria (Kumar et al. 2015). In the medical-industrial context, fermentation creates numerous biomaterials, such as exopolysaccharides used in the health industry (Kumar et al. 2015). The application of biotechnology in fermentation has grown at an unprecedented rate since the first discovery in as early as 6000 BC (Rahman 2013). From that time on, the impact of biotechnology in fermentation has evolved medical applications that are well beyond its more traditional uses.

Trillions of microbes live symbiotically in the intestines, and some play a vital role in human health (Rahman 2013). The microbiome contains bacteria that process nutrients and assist in stimulating enzymes required to increase the uptake of glucose, amino acids, and fatty acids (Rahman 2013). Recently, fermented products have been used to create and maintain a healthy gut microbiome (Rahman 2013). This paper investigates whether the claim that foods and dietary supplements created through fermentation foster the creation and maintenance of the human gut microbiome and assists in reducing the ailments of disease are supported by scientific evidence.

I have divided this paper into five sections: 1) a review of conventional fermentation methods, 2) a review of the medical claims on fermentation, 3) a review of the current uses of fermentation in medicine, 4) an analysis of the human gut microbiome, and 5) the assessment of current research and possible future uses of fermentation as it relates to the gut microbiome.

\section{Common Types of Fermentation}

Just as there are multitudes of fermentation products, there are numerous methods for their creation. Common types of fermentation include lactic acid fermentation, ethyl alcohol fermentation, and acetic acid fermentation. Each of these names refers to products generated from the anaerobic metabolism of organic molecules, especially carbohydrates, that uses carbon-rich molecules as the final electron acceptor following glycolysis.

Lactic acid fermentation (Figure 1) is generally used in preserving and producing a wide range of food items, including sauerkraut, pickles, and yogurts, through the action of lactic acid bacteria, such as lactobacilli (Steinkraus 1992). This process involves the conversion of a 3-carbon molecule, pyruvate, into a 3carbon molecule, lactic acid $\left(\mathrm{C}_{3} \mathrm{H}_{6} \mathrm{O}_{3}\right)$, and generates $\mathrm{NAD}^{+}$from $\mathrm{NADH}$ allowing ATP production to continue in low-oxygen environments (Wilkin and Akre 2019). The production of lactic acid kills other bacteria that can be found in the products (Wilkin and Akre 2019). While this type of fermentation is primarily used in food preparation, it may also confer antibiotic effects, such as the production of nisin, an antibiotic produced by Streptococcus lactis, which is active against gram-positive organisms, and hydrogen peroxide, which prevents food spoilage (Steinkraus 1992). This method is the most widely used method of fermentation for the preservation of food due to its heightened production of 
bacteriocins - antimicrobial proteins that protect foods from other bacteria - that render foods more resistant to microbial spoilage (Katz 2012).

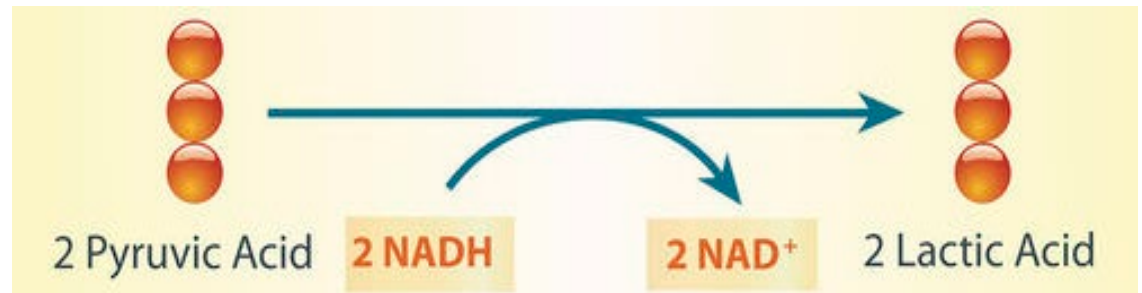

Figure 1. Lactic acid fermentation makes ATP in the absence of oxygen. This fermentation is conducted through the conversion of glucose to lactic acid with pyruvic acid as an intermediate. Each circle represents a carbon atom. Credit: Hana Zavadska, License: CC BY-NC 3.0 https://creativecommons.org/licenses/by-nc/3.0/

Another widely used form of fermentation is ethyl alcohol fermentation (Figure 2). This process is a form of alcohol fermentation, where yeast (generally varieties of Saccharomyces cerevisiae), when under anaerobic conditions, converts monosaccharides such as glucose to pyruvic acid, in turn converting pyruvic acid into ethanol $\left(\mathrm{C}_{2} \mathrm{H}_{5} \mathrm{OH}\right.$, Wilkin and Akre 2019). This process is mainly for the creation of biofuels and alcohol (beer and wine, Wilkin, and Akre 2019). While still used for preservation, it is most often useful for beverages and plant medicines such as tinctures (Katz 2012).

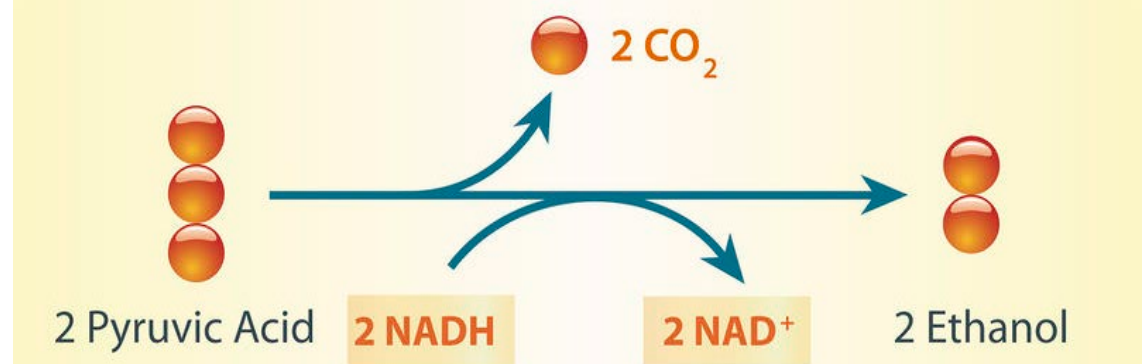

Figure 2. Ethyl alcohol fermentation makes ATP in the absence of oxygen. This method is conducted through the conversion of glucose to ethanol with pyruvic acid as an intermediate. Each circle represents a carbon atom. Credit: Hana Zavadska, License: CC BY-NC 3.0 https://creativecommons.org/licenses/by-nc/3.0/

Acetic acid fermentation, another commonly used method, takes ethyl alcohol fermentation one-step further with the use of acetic acid bacteria such as bacteria from the Acetobacteraceae family transforming ethanol into further compounds (Mamlouk and Gullo 2013). Through partial oxidation of carbohydrates, these bacteria release aldehydes, ketones, and organic acids to create products including vinegar, cocoa powder, palm wine, and kombucha 
(Mamlouk and Gullo 2013). This process is also used to make a range of industrial compounds such as organic surfactants, and pharmaceuticals such as therapeutic drugs for the treatment of non-insulin-dependent diabetes mellitus, and shikimate, an intermediate for several antibiotics (Mamlouk and Gullo 2013).

While this paper primarily discusses the consumption of fermented foods, there is an important distinction to make between fermentation in the body (in situ) and fermentation outside of the body (ex-situ). The most widely known in situ fermentation is lactic acid fermentation within the muscles. After periods of anaerobic exercise, such as sprinting, the muscles in the body undergo the breakdown of glucose in the muscle cells under limited oxygen availability causing a buildup of lactic acid (Wilkin and Akre 2019). Another type of in situ fermentation happens in the intestine where multitudes of distinct species, especially bacteria, that help the body to regulate homeostasis, known as the microbiota (Macfarlane and Macfarlane 2011). A part of this system includes the microbiome found within the large intestine where, in healthy individuals, short-chain fatty acids (acetate, propionate, and butyrate, among others) are produced through in situ fermentation (Macfarlane and Macfarlane 2011). This fermentation also produces intermediates such as lactate, and succinate, and phenolic compounds, such as phenol, p-cresol, and indole (Verbeke et al. 2015). Although essential for overall human health, these internal fermentation reactions are outside the scope of this paper. The products described throughout this paper are derived from external fermentation processes, ex-situ fermentation, though the chemical processes of fermentation are the same.

\section{Medical Claims on Fermentation}

Fermented foods have been lauded for extending shelf life and increasing their safety (Fernández et al. 2015, Rezac et al. 2018). These food items are staples of modern human diets. They include carbon dioxide in creams and butter (Adhikari et al. 2018), acetic acid in vinegar (Budak et al. 2014), lactic acid in dairy products (Burgain et al. 2014), ammonia in cheeses (Redruello et al. 2013), fatty acids found in hamburgers and French fries (Robledo de Dios et al. 2015), and many others.

There is a resurgence of interest in fermented foods due to its purported health benefits. Harvard University (Bilodeau 2018) and the British Broadcasting Corporation (BBC) (Lewin and Torrens 2018) and other organizations have claimed that fermented products provide benefits beyond essential nutrition. The claims of some health enthusiasts for fermented products include the reduction in risk of several metabolic and immune disorders, obesity, and infectious diseases (Lewin and Torrens 2018). However, the claims of the direct effect on human health of fermented foods have limited clinical support (Rezac et al. 2018).

Other benefits of fermentation for individual health state that it may create new compounds of medicinal significance. Products such as lactic acid, B vitamins, antioxidants, and short-chain fatty acids can be synthesized in large 
amounts, allowing for therapeutics and overall positive effects on the general health of a person (Marco et al. 2017). Also, it has been stated that the benefits of fermentation in human health includes the improvement of digestive health, immune system, and digestion (Coyle 2019). Accordingly, digestive health is aided by probiotics, live microorganisms with possible beneficial effects on the host, used during fermentation, as they can allegedly restore a balance of helpful bacteria in the gut microbiome (Barko et al. 2018). Fermented foods may also lessen symptoms of irritable bowel syndrome (IBS) and irritable bowel disease (IBD), diarrhea, bloating, and constipation due to digestive issues (Coyle 2019). As with digestive health, the probiotics from fermented food can boost the immune system to reduce the risk of infection and increase the speed of recovery when sick. As fermentation works to break down nutrients in food, food is allegedly easier to digest (Coyle 2019). This process may increase the absorption of beneficial nutrients, including potent biomolecules in small amounts, and allow them to be used more readily by the body.

Lactic acid has been shown to reduce the inflammatory cytokine secretion of bone-marrow-derived macrophages and dendritic cells (Marco et al. 2017). B vitamins are widely used within the medical and food industries as supplements for the health of the brain, nervous system, and immune system (Fang et al. 2017, Marco et al. 2017). These vitamins are produced through aerobic or anaerobic synthesis (Fang et al. 2017). Antioxidants from fermentation have also been claimed to be beneficial to human health (Gagnon et al. 2015, LaPointe and Roy 2015, Marco et al. 2017). Bacteria use these compounds to scavenge free oxygen radicals, which are believed to protect from and repair DNA damage (Gagnon et al. 2015). Short-chain fatty acids have been implicated in human health as a product of bacterial fermentation, where the lack of short-chain fatty acids was found to contribute to immune defects (Smith et al. 2013, Marco et al. 2017). This study assesses both gnotobiotic mice - those with carefully controlled populations of bacteria and viruses, and germ-free mice - those with no microorganisms at all (Williams 2014). When these groups are supplemented with short-chain fatty acids, their cTreg (colonic regulatory $\mathrm{T}$ cells which modulate immune response) and $\mathrm{CD}^{+} \mathrm{T}$ ( $\mathrm{T}$ helper cells that suppress or regulate immune responses) cell number increased (Smith et al. 2013, Campbell 2016, Zhu and Paul 2008). With each of these studies, researchers assess the impact of fermentation in medicine.

\section{Current Uses of Fermentation in Medicine}

The first use of fermentation in modern medicine was penicillin (Figure 3). This compound is the first antibiotic ever isolated and is still widely used today (Rahman 2013). Currently, the fermentation of penicillin is scaled to an industrial measure, which has increased the overall production of penicillin thousand-fold for use in general antibiotic treatments (Rahman 2013).

Chemical and pharmaceutical products that are medically significant are being increasingly produced using fermentation. These products include 
metabolites, enzymes, transformation products, or recombinant biomolecules, for instance, ethyl alcohol, L-glutaminase, and L-asparaginase for treatment of lymphocytic leukemia, steroids such as hydrocortisone, or luteinizing hormone for infertility (Rahman 2013). Based on these uses and current research, fermentation products are also now useful in treatments for infectious diseases, including a wide range of gastrointestinal infections, metabolic disorders, and immunological disorders, such as colitis and hyperglycemia (Corsello et al. 2017, Boulangé et al. 2016).

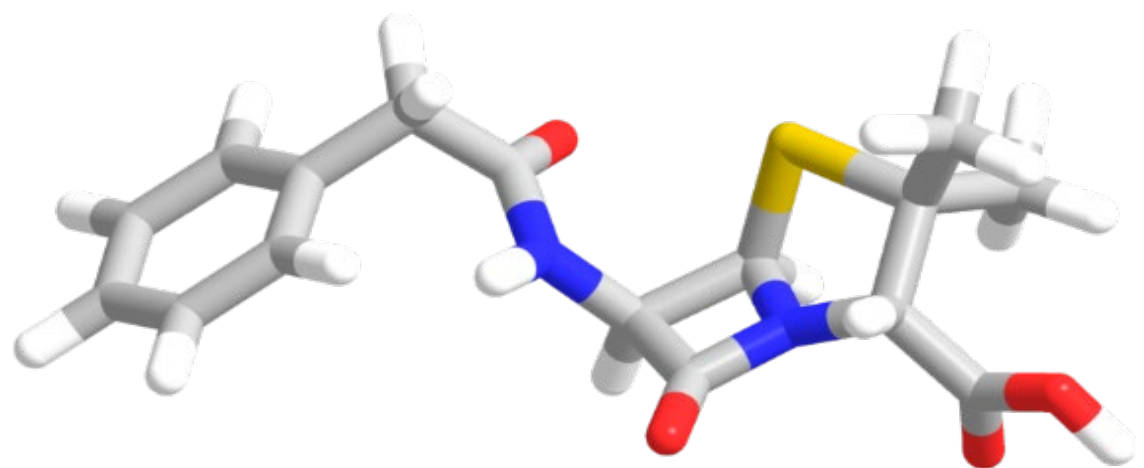

Figure 3. Chemical structure of Penicillin G. The sulfur and nitrogen of the thiazolidine ring are shown in yellow and blue, respectively. Oxygen molecules are shown in red, while the white depicts hydrogen molecules, https://commons.wikimedia.org/wiki/File:Penicillin-G 3D.png\#filelinks

Respiratory and gastrointestinal tract infections are common in young children. These infections, termed "common infectious diseases," include acute gastroenteritis, rhinitis, tracheitis, and laryngitis (Corsello et al. 2017). Through a multicenter, randomized controlled trial, it was found that dietary supplementation of milk fermented with Lactobacillus paracasei was effective in preventing common infectious diseases in young children (Corsello et al. 2017). Due to the microbial components of fermented foods, their probiotic strains can stimulate innate and acquired immune responses (Corsello et al. 2017). Milk fermented with L. paracasei was found to provide anti-inflammatory properties, which is caused by the stimulation of interleukin-10 (IL-10, Corsello et al. 2017). The fermented milk provided an immunostimulatory effect and a significant increase of both innate and acquired immunity peptides. Epithelial cells, including the Paneth cells located in the small intestine, and neutrophils produce innate immunity peptides. The peptides of acquired immunity play a role in defending the body against numerous pathogens and regulate $T$ cells, macrophages, and monocytes. The peptides of acquired immunity play a role in local immunity as the first line of defense in the mucosae and regulate gut microbiota composition, which drives the communication between gut bacteria and the host immune 
system (Corsello et al. 2017). With the production of the immune peptides, it is evident that the fermentation product of milk and L. paracasei is a viable strategy for preventing some infectious diseases.

In metabolic and immunological disorders, molecular interactions in the symbiotic gut microbiota influence energy metabolism, lipid accumulation, and immunity. Changes in the gut microbiome have been correlated with an increase in metabolic and immune disorders. The transient changes in the balanced microbial ecosystems of the gut microbiome lead to the disruption of this relationship, a phenomenon termed dysbiosis (Boulangé et al. 2016). Fermentation products, in the form of food or dietary supplements, can act as immune-stimulating factors to increase the production of short-chain fatty acids while decreasing inflammation.

Human and other animal studies have shown that the composition of the gut microbiome can be influenced through the diet. The Actinobacteria, Bacteroidetes, Firmicutes, Proteobacteria, and Verrucomicrobia are among major taxonomic groups of bacteria being studied, and the specific and relative species composition of each of these groups can influence animal metabolism, energy balance, gut permeability, and inflammation in humans and other animals (Wen and Duffy 2017). In a mouse model obesity study, the relationship between diet, gut microbial composition, and energy balance was investigated concerning a prototypic Western diet consisting of high-fat and high- monosaccharide content or a standard diet of low-fat and polysaccharide-rich content (Turnbaugh et al. 2008). This research found that diet-induced obesity caused growth of bacteria placed in a single clade in the class Mollicutes (Firmicutes), becoming the dominant group of microbes in the distal gut microbiome, where it is typically found in low abundance in samples (Turnbaugh et al. 2008). When a sample of the microbiota was transplanted from the Dietary-Induced Obese mice to lean mice, the recipients' adiposity increased. These researchers also found that this surge might be controlled by dietary manipulations, using gnotobiotic models (mice with carefully controlled microbiota) and comparative metagenomics (Turnbaugh et al. 2008). Comparative metagenomics aims to study the diversity of microbes through large-scale sequencing of microbial communities; in the case of this study, the communities studied were part of the gut microbiota (Huson et al. 2009, Turnbaugh et al. 2008). Similar studies conducted in children and adults have shown correlations between gut microbe composition and diseases, such as type-2 diabetes, obesity, IBS, and inflammation (Lambert et al. 2015, Durack and Lynch 2019, Jayakumar and Loomba 2019, Cooper et al. 2017).

The study of the human gut microbiome has increasingly become a topic of interest for researchers worldwide, including the effects of fermented products on the composition of the gut microbiome. Gut microbiota can be correlated to IBDs such as Crohn's Disease (CD), and other diseases such as Non-Alcoholic Fatty Liver Disease, and diabetes (Lambert et al. 2015, Durack and Lynch 2019, Jayakumar and Loomba 2019). Dysbiosis is increasingly being implicated in 
varying forms of IBD, where the loss of enteric bacterial diversity and an expansion in the relative abundance of family Enterobacteriaceae has been associated with the onset of CD (Durack and Lynch 2019).

\section{The Human Gut Microbiome}

The human gastrointestinal microbiome is a diverse collection of organisms, containing Bacteria, Archaea, Fungi, as well as viruses, and a diverse array of eukaryotic organisms that have traditionally been "protists." These organisms are implicated in a wide range of physiological processes vital to the host's health, such as homeostasis, metabolism, immunologic activity, and neurobehavioral development. Many of these organisms are considered essential to the life and health of mammals. The microbiome is dynamic and subject to significant changes over the lifetime of the host (Mills et al. 2019).

The gut microbiome is formed after the birth of a mammal when it meets the bacteria located in the mother's birth canal (or vagina) and the environment. Once biologically established, the composition and configuration of the microbiome begins to develop into a mature and functional unit (Barko et al. 2018). The first three years of life for the child are crucial for developing a healthy gut microbiome through food and environmental exposure to proper organisms. Each change in the stage of life, for example, when an infant is weaned from breastfeeding and moved to solid foods, is accompanied by a shift in the gut microbiome. These changes allow the gut microbiome to adapt and assist the body in processing sources such as complex carbohydrates, xenobiotics, or substances foreign to the organism, and participate in vitamin synthesis through the culturing of beneficial bacterial species within the gut (Barko et al. 2018).

Figure 4 depicts some of the numerous factors that may affect the health and composition of the gut microbiome. Initially, the essentially sterile neonatal microbiome begins colonization through maternal microflora and environmental bacteria during birth, leading into the perinatal and infant periods (Barko et al. 2018). From the perinatal and infant stages, the gut microbiota, through diet, genetics, immunity, sanitation, feeding regimen, and environmental microbes, can become healthy and mature (Barko et al. 2018, Mills et al. 2019). Individuals in this stage can also experience persistent dysbiosis due to antibiotics, malnutrition, or infection, where the microbiota becomes simple, has low diversity, and becomes permissive to pathogens. If treated, microbiomes afflicted with persistent dysbiosis can be recovered into a healthy, mature microbiome. In cases where persistent dysbiosis is not treated, infants can experience transient dysbiosis through acute or chronic disturbance. The healthy mature state, transient dysbiosis state, and persistent dysbiosis state can change through treatments, acute disturbances, or chronic disturbances (Barko et al. 2018).

Dysbiosis in the gut microbiome, which can be attributed to numerous factors, occurs when there is an imbalance of commensal and pathogenic bacteria in the gut microbiome. This imbalance can be caused by taking antibiotics, 
malnutrition, or from infectious states from pathogens, such as Norovirus, or the bacterium, Vibrio cholerae, the etiological agent of cholera (Kellemayer 2019).

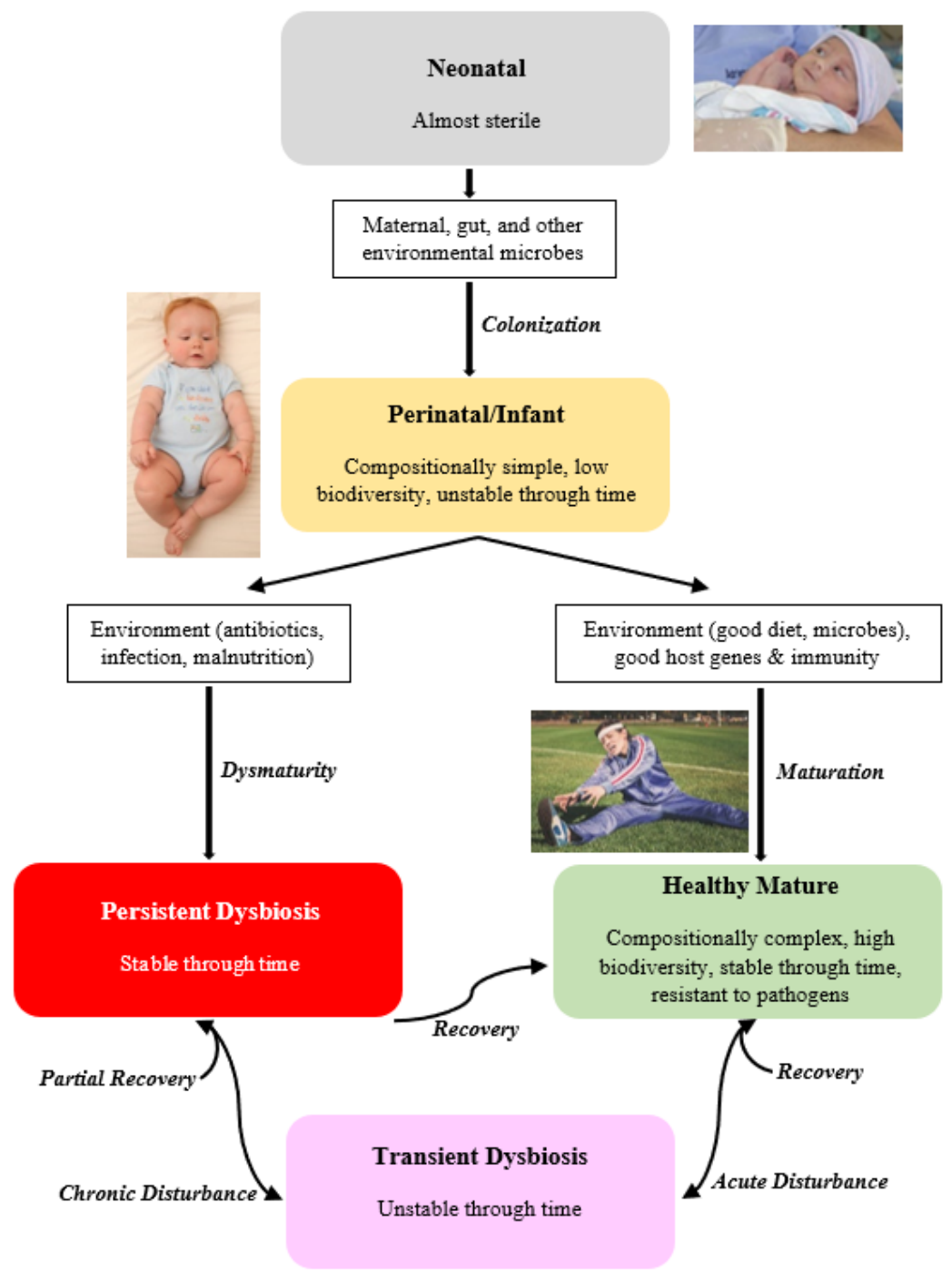

Figure 4. Some factors influencing the gut microbiome over time. While the adult microbiome tends to be stable, several factors influence the steady states of the gut microbiome at birth and in disease (Barko et al. 2018). In contrast to a healthy, adult, microbiome dysbioses, whether transient (or temporary) or persistent are characterized by being compositionally simple, having high biodiversity, as well as being stable through time and resistant to pathogens. This figure has been modified from https://www.ncbi.nlm.nih.gov/pmc/articles/PMC5787212/ 
While it is widely understood that the microorganisms in the gastrointestinal tract assist the body in breaking down food particles for energy, other functions of the gut microbiome are not understood. The interaction between the gut microbial communities, the gastrointestinal tract, and the central nervous system (CNS) are recognized as the microbiota-gut-brain axis, which is subject to increasing scientific inquiry (Guernier et al. 2017). This axis operates through various mechanisms, including neural, hormonal, and immunological pathways. Previous investigations (Al-Asmakh et al. 2012, Rhee et al. 2009, Chen et al. 2013) have demonstrated the microbiota-gut-brain axis plays a crucial role in the development, maturation, and function of the brain, where a disruption in the gut microbial community can affect behavior and brain systems (Guernier et al. 2017).

The microbiota-gut-brain axis influences the CNS directly through the vagus nerve, a nerve of the autonomic nervous system that regulates the gut (Yang et al. 2016). Bacteria in the microbiome work to produce peptides and cytokines for activating this nerve resulting in signals being sent to the CNS that affect behavior, efferent neural activity, and inflammation (Yang et al. 2016). Dysbiosis, a gut microbiome disruption or imbalance, is implicated in altered phenotypes, such as human brain diseases, including anxiety, depression, and chronic pain (Guernier et al. 2017).

Figure 5 depicts a general overview comparing a balanced microbiota to microbial dysbiosis in the gut, concerning an outside influence causing dysbiosis (soil-transmitted helminths, Guernier et al. 2017). Helminths are parasitic worms that cause an imbalance of the gut-brain axis, leading to possible detriment to brain development as well as a nutritional deficit, anemia, and reduced growth (Guernier et al. 2017). As the gut microbiota matures over the first three years of life, it coincides with the critical window of early brain development. During this development, nutrition, infection, the environment, or stress can affect this development into adulthood (Guernier et al. 2017). As the microbiome develops, it plays a pivotal role in systems regulating the CNS, including synaptogenesis, or the development of connections between neurons, and myelination, or the development of the proteinaceous and lipid cover surrounding many neurons (Guernier et al. 2017). This bidirectional functional communication axis between the gut and the central nervous system shows the importance of developing a healthy gut microbiome from a young age.

On Figure 4, the image of a newborn baby, which is part of a much larger image, comes from https://commons.wikimedia.org/wiki/File:Neonatal Nurse Practitioner.J and it is licensed under the Creative Commons Attribution-Share Alike 4.0 International license. The image of an infant comes from https://upload.wikimedia.org/wikipedia/commons/2/26/Baby in an infant bodysuit.jpg and it has been released into the public domain by its author, Cphilp at English Wikipedia. The image of an adult comes from https://commons.wikimedia.org/wiki/File:Healthy-person-woman-sport-1_(23698632413).jpg . and it is available under the Creative Commons CC0 1.0 Universal Public Domain Dedication. 


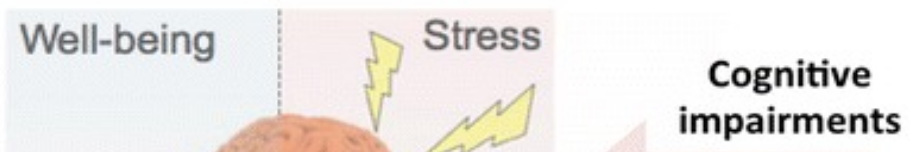

(2)
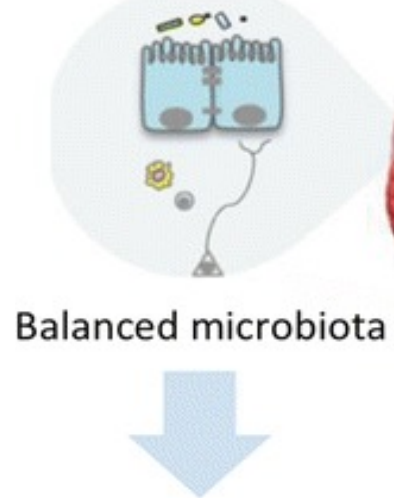

Homeostasis

(1)

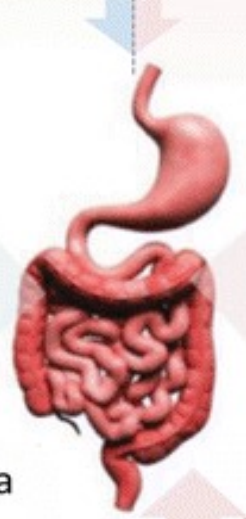

(3)

Dysbiosis
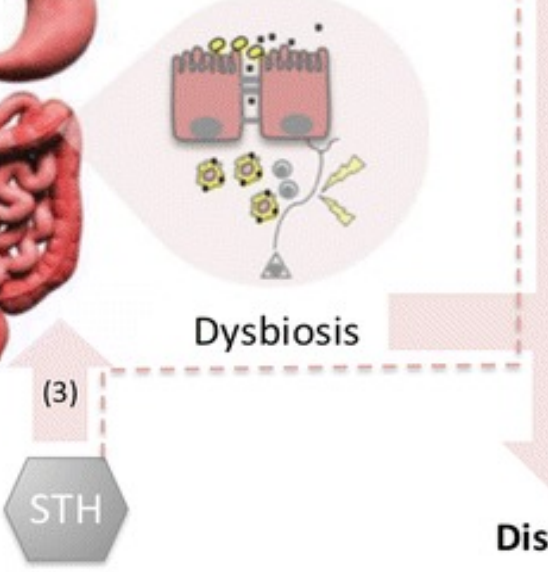

Disease

Figure 5. Effects of a healthy gut microbiota compared to the effects of a dysbiotic gut microbiota. The bidirectional access between the gut microbiota and the brain (1) denotes the link between disease, cognitive impairment, and dysbiosis in the gut microbiome (2) caused, in this case, by a soil-transmitted helminth (worm) infection (3, Guernier et al. 2017). $\quad$ https://bmcinfectdis.biomedcentral.com/articles/10.1186/s12879-016-2146-2 Open access article distributed under the terms of the Creative Commons AttributionInternational License. Open Access This article is distributed under the terms of the Creative Commons Attribution 4.0 International License (http://creativecommons.org/licenses/by/4.0/), which permits unrestricted use, distribution, and reproduction in any medium, provided you give appropriate credit to the original author(s) and the source, provide a link to the Creative Commons license, and indicate if changes were made. The Creative Commons Public Domain Dedication waiver (http://creativecommons.org/publicdomain/zero/1.0/) applies to the data made available in this article, unless otherwise stated. 
The gut microbiome also plays a pivotal role in neurological development (Barko et al. 2018). Numerous developmental disorders, such as anxiety and depression into adulthood, have been linked to a poor gastrointestinal microbiome (Yang et al. 2016). Compositional and functional deviances from a healthy gut microbiome are associated with abnormal development in the form of atopy or the genetic tendency to form allergies, obesity, and autism spectrum disorder (Barko et al. 2018). As the human gut is home to as many as 100 trillion cells, the complex, profoundly diverse conglomerations provide enzymatic capabilities that play a fundamental role in host physiology (Yang and Kweon 2016). This community of microorganisms contributes to host physiology through the increase of energy availability, production of acetate, and large amounts of numerous other metabolites to be used by the host (Yang and Kweon 2016). While the gut microbiota is an exciting emerging topic, further studies are needed to understand the intricacies and implications in human health and disease.

\section{Assessment of the Current Research and Possible Future Directions of Fermentation in the Gut Microbiome}

Currently, there are numerous fermentation therapies available that administer prebiotics, or nutrients that promote microbial growth, and probiotics, or living microbes, as well as fecal transplantation (Barko et al. 2018). These treatments are meant to alter the microbial community of the gastrointestinal microbiome to improve overall health.

Prebiotics are compounds that cannot be processed directly by the human body and are only made digestible through the fermentation of short-chain carbohydrates, namely oligosaccharides. Bacteria in the genus Bifidobacterium and several species of Lactobacillus, including L. rhamnosus, L. reuteri, and $L$. fermentum, are responsible for the fermentation of those prebiotics (Cummings et al. 2001, Davani-Davari et al. 2019). These compounds are fermented into shortchain fatty acids that provide essential nutrients, lower the $\mathrm{pH}$ of the gastrointestinal lumen, and induce anti-inflammatory responses in the distal gut (Barko et al. 2018). In contrast, probiotics are formulations of live organisms that reduce intestinal permeability and increase secretion of defensins - cysteine-rich, cationic proteins acting as host defense peptides (Machado and Ottolini 2015). Also, they prevent pathogen colonization, produce short-chain fatty acids, stimulate IgA - a major serum immunoglobulin acting as the predominant antibody protecting mucosal surfaces (Woof and Kerr 2006) - and promote resistance against pathogens (Barko et al. 2018). Probiotic strains, such as Bifidobacterium spp. and Lactobacillus spp., are implicated in this in situ type of fermentation (van Zanten et al. 2012). There are several beneficial effects shown in human intervention studies, including reducing the severity of dermatitis and preserving insulin sensitivity (van Zanten et al. 2012). 
The combination of prebiotics and probiotics is termed as synbiotics ${ }^{3}$, and patients are predicted to gain the beneficial health effects of both (Schrezenmeir and de Vrese 2001, De Vrese and Schrezenmeir 2008, van Zanten et al. 2012). In vitro and post-operational clinical studies have shown that synbiotics have resulted in a favorable shift in cancer biomarkers and a lower incidence of infection following an operation. Some synbiotics have also been observed to increase the levels of species of Bifidobacterium and Lactobacillus (van Zanten et al. 2012).

Other studies have investigated the benefits of synbiotic treatments in animal models and humans for their effects on gut microbiota, metabolic syndromes, and endotoxin levels. In a randomized, double-blind controlled trial, the effects of a starter formula, an infant formula used in place of traditional breastfeeding, containing synbiotic supplements fed to infants born to HIV+ mothers through cesarean delivery used to investigate the possibility of improving their microbiome composition (Cooper et al. 2017). This study was conducted with the assumed knowledge that infant guts at birth are sterile, but quickly acquire colonizing microbes through delivery. It was determined that children born via cesarean delivery had a 10-day delay in colonizing bifidobacteria and lactobacilli as compared to the two to three-day colonization period of vaginally delivered infants (Cooper et al. 2017). Further research on the development of the gut microbiota has introduced the possibility of in utero colonization due to the presence of microorganisms in the meconium, or the first excrement in mammalian infants, and the amniotic fluid. However, there is insufficient evidence currently supporting this claim due to the lack of proper contamination controls and evidence of bacterial viability (Mills et al. 2019).

The postnatal colonization of the microbiome is also dependent on the feeding method. Breastfed children are more likely to gain the necessary microbiota faster than formula-fed children because maternal human milk contains bioactive components, including undigestible oligosaccharides that stimulate bifidobacterial growth (Cooper et al. 2017). The 362 infants in this study were born to HIV+ mothers, who elected to formula feed from birth, allowing the investigation of a starter formula supplemented with a mixture of bovine milk-derived oligosaccharides and $B$. animalis subspecies lactis strain CNCM I-3446 (Cooper et al. 2017). The test formula safely, as determined by anthropometric measurements (weight, recumbent length, and head circumference), corrected the low bifidobacteria levels of the gut microbiota, lowered the $\mathrm{pH}$ of the infant fecal matter, and improved its microbial composition

\footnotetext{
${ }^{3}$ In English and in other languages, the placement of an " $m$ " and not an " $\mathrm{n}$ " is generally considered grammatically correct. In the case of this paper, the word "synbiotic" alludes to "synergism" and it is reserved for products where the prebiotic compound selectively favors the probiotic species (Schrezenmeir and de Vrese 2001, Pandey et al. 2015). This concept was first coined by Glenn Gibson who investigated the benefits of combining prebiotics and probiotics.
} 
(Cooper et al. 2017) highlighting the capabilities of probiotics and prebiotics for use in gut microbiota development.

Randomized control trials have also been conducted to assess the effects of synbiotics on metabolic syndrome. These studies involve several associated physiological, biochemical, clinical, and metabolic factors that increase the risk of diseases, including Type 2 Diabetes Myelitis and all-cause mortality, with risk factors of high waist circumference, hypertension, and insulin resistance and constipation (Myers et al. 2019). Mohammadi-Sartang et al. (2018) studied metabolic conditions in adults with obesity; subjects consumed fortified yogurt containing prebiotic fiber and probiotic cultures. They determined, through body composition and biochemical indices, that the consumption of the fortified yogurt resulted in a significant reduction in body fat mass, body fat percentage. This study also found that fortified yogurt containing fiber and probiotic cultures also significantly increased high density lipoprotein-cholesterol (HDL) levels and insulin sensitivity, among other benefits. While this study provides some insights on the use and benefits of probiotics, further research is needed to isolate the mechanisms and efficacy of the use of yogurt inundated with synbiotics as a preventative strategy for metabolic syndrome in obese individuals (MohammadiSartang et al. 2018).

Synbiotics have been shown to be somewhat useful in curbing metabolic syndrome and weight gain in obese adults and have also been shown to improve the quality of life for those suffering from functional constipation. An early study on yogurt containing polydextrose, L. acidophilus NCFM $^{4}$, and B. lactis HN019 found that the synbiotics in this yogurt had a significant reduction in the colonic transit time over the control group yogurt (Magro et al. 2014). However, in a more recent randomized, double-blind, placebo-controlled study using similar synbiotics with the same strains of probiotics to adjust the gastrointestinal microbiota, subjects were evaluated according to the Bristol stool scale and multiple patient assessments (Lim et al. 2018). While the results of this study showed a reduction of severity of symptoms, the differences between the control and test groups were not statistically significant. Although these results are inconclusive for the overall effects of the synbiotic supplements, they still show promise for more research in the future with larger test groups.

An exploratory study conducted on novice triathletes of prebiotics and probiotics investigated interventional methods on endotoxin levels found in the gastrointestinal tract (Lim et al. 2018). This study used a multi-strain probiotic, prebiotic, and antioxidant study consisting of multiple strains of bacteria in the genera Lactobacillus and Bifidobacterium as well as fructooligosaccharides, inulin, pectin, $\alpha$-lipoic acid, $\mathrm{N}$-acetyl-carnitine, vitamin $\mathrm{C}$, quercetin, resveratrol, and curcumin were provided in a randomized, repeated-measure, double-blind, placebo-controlled study. This study was meant to determine their efficacy in

\footnotetext{
${ }^{4}$ The codes NCFM and HN019 next to B. lactis, refer to strains of these species of bacterium.
} 
reducing gastrointestinal complaints of novice athletes in training. Roberts et al. (2016) found that the symptoms associated with gastrointestinal distress, such as cramping, nausea, and abdominal pain, were reduced, and the multi-strain treatment may provide support for minimizing these symptoms. While this study appears to show promise for the future of prebiotics and probiotics in athletics, it also states the benefits are likely strain specific. Further studies on specific strains and benefits provided are required to provide support to the claims of prebiotic and probiotic interventions.

Fecal transplantation is an emerging practice involving the transfer of feces from a healthy gut microbiome to the intestines of a diseased recipient. This technology has shown success in treating Clostridium difficile infections with an approximately $90 \%$ cure rate (Kellermayer 2019, Barko et al. 2018). Though the benefit of this practice is not yet attributable directly to the effects of fecal transplantation, further research could allow for the discovery of the cause and the synthesis of the necessary compounds (Kellermayer 2019). The possible causes of the benefit include the transfer of commensals, viruses, proteins, vitamins, or numerous other substances not yet identified (Barko et al. 2018). While the cause of the acquired benefits is not currently known, this technology can be studied further to enhance and improve the knowledge of the conditions within the gastrointestinal microbiome.

A double-blind, randomized study of fecal microbiota transplantation was conducted to determine the efficacy and safety of this treatment for patients with ulcerative colitis. These patients experienced mildly to moderately active ulcerative colitis and underwent fecal transplantation. The test group was administered feces from healthy donors, and the control group received autologous, or from themselves, fecal microbiota (Rossen et al. 2015). Phase two trials yielded no significant differences between the control and the test groups, though this is possibly attributed to the limited number of subjects undergoing this treatment (Rossen et al. 2015). While there was no statistically significant improvement, future studies can elucidate the distinct differences in the microbiota of individuals who did and did not respond to treatment (Rossen et al. 2015). In addition, future studies should consider the amount and composition of fecal matter from healthy donors to determine the best avenues for treatment using fecal matter transplantation.

Further studies in fecal transplantation for amyotrophic lateral sclerosis (ALS) have been proposed through multicenter randomized, double-blind clinical trials (Mandrioli et al. 2019). This study expects that fecal matter transplantation can increase $\mathrm{T}$ regulatory lymphocyte count allowing the immune system to surround the motoneurons to become anti-inflammatory and neuroprotective through the microbiota-gut-brain axis. This trial is set to be the first to use fecal matter transplantation as an intervention for ALS and early disease progression. This study may provide more insight into the future applications of fecal matter 
transplantation, as well as information on the mechanisms of the microbiota-gutbrain axis relating to immunity.

\section{Conclusions and Future Research Directions}

The current tested uses of fermented products in medicine are few but they appear to be versatile in their capabilities against some infections and diseases. This adaptability can facilitate further research and development in medicine. Some fermented food products have been shown to benefit overall health, even though they cannot be touted as a complete cure. Consuming fermented products, including synbiotics, fortified yogurts, and general fermented foods may become a widely used addition in treatment plans aiming to improve the general health of an individual.

Further research is needed to understand the mechanisms that cause changes in the human gut microbiome to treat and support atypical systems or systems experiencing dysbiosis. Studies need to be conducted on metabolic pathways of the immune responses, as well as the intake and use of prebiotics and probiotics. Metagenomics can profile and assist in understanding the gut microbiome in humans. This approach can assist in predicting disease relapse and the possibility of chronicity (Aziz et al. 2013). Recent studies of gnotobiotic animals, with prebiotics, probiotics, and antibiotics alone or in combination may provide a method to understand and manage clinical symptoms, syndromes, and disorders. Future areas of research include the identification of disease susceptibility, effects of the microbiome on pharmacology, mechanistic studies of molecular mechanisms, and confirmatory human studies for individualized therapeutics (Arora and Bäckhed 2016). The future of fermentation in the gut microbiome has extensive possibilities for research and applications in medicine.

\section{Acknowledgments}

Five reviewers offered comments that improved this paper. To them, my genuine thanks. My thanks as well to readers who offered improvements in the grammar.

\section{Literature Cited}

Adhikari, B. M., T. Truong, N. Bansal, and B. Bhandari. 2018. Use of gases in dairy manufacturing: A review. Critical Reviews in Food Science and Nutrition 58(15):2557-2569. https://doi.org/10.1080/10408398.2017.1333488

Al-Asmakh, M., F. Anuar, F. Zadjali, J. Rafter, and S. Pettersson. 2012. Gut microbial communities modulating brain development and function. Gut Microbes 3(4):366-373. https://doi.org/10.4161/gmic. 21287

Arora, T. and F. Bäckhed. 2016. The gut microbiota and metabolic disease: current understanding and future perspectives. Journal of Internal Medicine 280(4):339-349. https://doi.org/10.1111/joim.12508

Aziz, Q., J. Doré, A. Emmanuel, F. Guarner, and E. M. Quigley. 2013. Gut microbiota and gastrointestinal health: current concepts and future directions. Neurogastroenterology \& Motility 25(1):4-15. https://doi.org/10.1111/nmo.12046

Barko, P. C., M. A. McMichael, K. S. Swanson, and D. A. Williams. 2018. The gastrointestinal microbiome: A review. Journal of Veterinary Internal Medicine 32(1):9-25. https://doi.org/10.1111/jvim.14875 
Bilodeau, K. 2018 (May 16). Fermented foods for better gut health. Retrieved from Harvard Health Publishing. https://www.health.harvard.edu/blog/fermented-foods-for-better-gut-health2018051613841

Boulangé, C. L., A. L. Neves, J. Chilloux, J. K. Nicholson, and M. E. Dumas. 2016. Impact of the gut microbiota on inflammation, obesity, and metabolic disease. Genome Medicine 8(1):42. https://doi.org/10.1186/s13073-016-0303-2

Budak, N. H., E. Aykin, A. C. Seydim, A. K. Greene, and Z. B. Guzel-Seydim. 2014. Functional properties of vinegar. Journal of Food Science 79(5):R757-R764. https://doi.org/10.1111/1750$\underline{3841.12434}$

Burgain, J., J. Scher, G. Francius, F. Borges, M. Corgneau, A. M. Revol-Junelles, C. Cailliez-Grimal, and C. Gaiani. 2014. Lactic acid bacteria in dairy food: surface characterization and interactions with food matrix components. Advances in Colloid and Interface Science 213:21-35. https://doi.org/10.1016/j.cis.2014.09.005

Campbell, D. J. 2015. Control of regulatory T cell migration, function, and homeostasis. The Journal of Immunology 195(6):2507-2513. https://doi.org/10.4049/jimmunol.1500801

Capalbo, D. M. 1995. Bacillus thuringiensis: Fermentation profcess and risk assessment. A short review. Memórias do Instituto Oswaldo Cruz 90(1):135-8. https://doi.org/10.1590/S0074$\underline{02761995000100026}$

Chen, X., R. D'Souza, and S. T. Hong. 2013. The role of gut microbiota in the gut-brain axis: current challenges and perspectives. Protein \& Cell 4(6):403-414. https://doi.org/10.1007/s13238-013$\underline{3017-\mathrm{X}}$

Cooper, P., K. D. Bolton, S. Velaphi, N. de Groot, S. Emady-Azar, S. Pecquet, and P. Steenhout. 2017. Early benefits of a starter formula enriched in prebiotics and probiotics on the gut microbiota of healthy infants born to HIV+ mothers: a randomized double-blind controlled trial. Clinical Medicine Insights: Pediatrics 10:119-130. https://doi.org/10.4137/CMPed.S40134

Corsello, G., M. Carta, R. Marinello, M. Picca, G. De Marco, M. Micillo, D. Ferrara, P. Vigneri, G. Cecere, P. Ferri, P. Roggero, G. Bedogni, F. Mosca, L. Paparo, R. Nocerino, and R. Berni Canani. 2017. Preventive effect of cow's milk fermented with Lactobacillus paracasei CBA L74 on common infectious diseases in children: a multicenter randomized controlled trial. Nutrients 9(7)669. https://doi.org/10.3390/nu9070669

Coyle, D. 2019 (January 15). What is Fermentation? The lowdown on fermented foods. Healthline. https://www.healthline.com/nutrition/fermentation

Cummings, J. H., G. T. Macfarlane, and H. N. Englyst. 2001. Prebiotic digestion and fermentation. The American Journal of Clinical Nutrition 73(2):415-420. https://doi.org/10.1093/ajcn/73.2.415s

Davani-Davari, D., M. Negahdaripour, I. Karimzadeh, M. Seifan, M. Mohkam, S. J. Masoumi, A. Berenjian, and Y. Ghasemi. 2019. Prebiotics: definition, types, sources, mechanisms, and clinical applications. Foods (Basel, Switzerland) 8(3):92. https://doi.org/10.3390/foods8030092

De Vrese, M. and J. Schrezenmeir. 2008. Probiotics, Prebiotics, and Synbiotics. Advances in Biochemical Engineering/Biotechnology 111:1-66. https://doi.org/10.1007/10_2008_097.

Durack, J. and S. V. Lynch. 2019. The gut microbiome: Relationships with disease and opportunities for therapy. The Journal of Experimental Medicine 216(1):20-40. https://doi.org/10.1084/jem.20180448

Fang, H., J. Kang, and D. Zhang. 2017. Microbial production of vitamin B12: a review and future perspectives. Microbial Cell Factories 16(1):15. http://doi.org/10.1186/s12934-017-0631-y

Fernández, M., J. H. Hudson, K. Korpela, and C. G. de los Reyes-Gavilán. 2015. Impact on human health of microorganisms present in fermented dairy products: an overview. BioMed Research International 2015(Article ID 412714):13 pp. http://dx.doi.org/10.1155/2015/412714

Gagnon, M., P. Savard, A. Rivière, G. LaPointe, and D. Roy. 2015. Bioaccessible antioxidants in milk fermented by Bifidobacterium longum subsp. longum strains. BioMed Research International 2015(Article ID 169381):12 pp. http://doi.org/10.1155/2015/169381

Guernier, V., B. Brennan, L. Yakob, G. Milinovich, A. C. Clements, and R. J. Soares Magalhaes. 2017. Gut microbiota disturbance during helminth infection: can it affect cognition and behaviour of children? BMC Infectious Diseases 17(1):58. http://doi.org/10.1186/s12879-016$\underline{2146-2}$ 
Huson, D. H., D. C. Richter, S. Mitra, A. F. Auch, and S. C. Schuster. 2009. Methods for comparative metagenomics. BMC Bioinformatics 10(S12). https://doi.org/10.1186/1471-2105-10-S1-S12

International Service for the Acquisition of Agri-biotech Applications (ISAAA). 2006. https://www.isaaa.org/resources/publications/pocketk/20/default.asp

Jayakumar, S., and R. Loomba. 2019. Review article: emerging role of the gut microbiome in the progression of non-alcoholic fatty liver disease and potential therapeutic implications. Alimentary Pharmacology \& Therapeutics 50(2):144-158. https://doi.org/10.1111/apt.15314

Katz, S. (Foreword by Pollan, M.) 2012. The Art of Fermentation: An In-depth Exploration of Essential Concepts and Processes from Around the World. Chelsea Green Publishing. White River Junction, Vermont, USA. 498 pp.

Kellermayer, R. 2019. Fecal microbiota transplantation: great potential with many challenges. Translational Gastroenterology and Hepatology 4:40. https://doi.org/10.21037/tgh.2019.05.10

Kumar Gupta, G., S. De, A. Franco, A. M. Balu, and R. Luque. 2015. Sustainable biomaterials: current $\begin{array}{llll}\text { trends, challenges and } & \text { Molecules }\end{array}$ https://doi.org/10.3390/molecules 21010048

Lambert, J. E., J. A. Parnell, B. Eksteen, M. Raman, M. R. Bomhof, K. P. Rioux, and R. A. Reimer. 2015. Gut microbiota manipulation with prebiotics in patients with non-alcoholic fatty liver disease: a randomized controlled trial protocol. BMC Gastroenterology 15:169. https://doi.org/10.1186/s12876-015-0400-5

Lewin, J. and K. Torrens. 2018 (October 1). The health benefits of fermenting. Retrieved from $B B C$ Good Food. https://www.bbcgoodfood.com/howto/guide/health-benefits-offermenting

Lim, Y. J., R. Jamaluddin, A. S. Hazizi, and J. Y. Chieng. 2018. Effects of synbiotics among constipated adults in Serdang, Selangor, Malaysia - a randomised, double-blind, placebocontrolled trial. Nutrients 10(7):824. https://doi.org/10.3390/nu10070824

Macfarlane, G. and S. Macfarlane. 2011. Fermentation in the human large intestine its physiologic consequences and the potential contribution of prebiotics. Journal of Clinical Gastroenterology 45:S120-S127. https://doi.org/10.1097/MCG.0b013e31822fecfe

Machado, L. R. and B. Ottolini. 2015. An evolutionary history of defensins: a role for copy number variation in maximizing host innate and adaptive immune responses. Frontiers in Immunology 6:115. https://doi.org/10.3389/fimmu.2015.00115

Magro, D. O., L. M. de Oliveira, I. Bernasconi, M. Ruela, L. Credidio, I. K. Barcelos, and C. S. Coy. 2014. Effect of yogurt containing polydextrose, Lactobacillus acidophilus NCFM and Bifidobacterium lactis HN019: a randomized, double-blind, controlled study in chronic constipation. Nutrition Journal 13:75. https://doi.org/10.1186/1475-2891-13-75

Mamlouk, D. and M. Gullo. 2013. Acetic acid bacteria: physiology and carbon sources oxidation. Indian Journal of Microbiology 53:377-384. https://doi.org/10.1007/s12088-013-0414-z

Mandrioli, J., A. G. Amedei, G. Cammarota, E. Niccolai, E. Zucchi, R. D'Amico, and L. Masucci. 2019. FETR-ALS Study Protocol: a randomized clinical trial of fecal microbiota transplantation in amyotrophic lateral sclerosis. Frontiers in Neurology 10:1021. https://doi.org/10.3389/fneur.2019.01021

Marco, M., D. Heeney, S. Binda, C. Cifelli, P. Cotter, B. Foligné, M. Gänzle, R. Kort, G. Pasin, A. Pihlanto, E. J. Smid, and R. Hutkins.. 2017. Health benefits of fermented foods: microbiota and beyond. Current Opinion in Biotechnology 44:94-102. https://doi.org/10.1016/j.copbio.2016.11.010

Mills, S., C. Stanton, J. A. Lane, G. J. Smith, and R. P. Ross. 2019. Precision nutrition and the microbiome, Part I: Current state of the science. Nutrients 11(4):923. https://doi.org/10.3390/nu11040923

Mohammadi-Sartang, M., N. Bellissimo, J. T. de Zepetnek, N. R. Brett, S. M. Mazloomi, M. Fararouie, and Z. Mazloom. 2018. The effect of daily fortified yogurt consumption on weight loss in adults with metabolic syndrome: A 10-week randomized controlled trial. Nutrition, Metabolism and Cardiovascular Diseases 28(6):565-574. https://doi.org/10.1016/j.numecd.2018.03.001

Myers, J., P. Kokkinos, and E. Nyelin. 2019. Physical activity, cardiorespiratory fitness, and the metabolic syndrome. Nutrients 11(7):1652. https://doi.org/10.3390/nu11071652 
Pandey, K. R., S. R. Naik, and B. V. Vakil. 2015. Probiotics, prebiotics and synbiotics - a review. Journal of Food Science and Technology 52(12):7577-7587. https://doi.org/10.1007/s13197015-1921-1

Rahman, M. 2013. Medical applications of fermentation technology. Advanced Materials Research 810:127-157. https://doi.org/10.4028/www.scientific.net/AMR.810.127

Redruello, B., V. Ladero, I. Cuesta, J. R. Álvarez-Buylla, M. C. Martín, M. Fernández, and M. A. Alvarez. 2013. A fast, reliable, ultra high performance liquid chromatography method for the simultaneous determination of amino acids, biogenic amines and ammonium ions in cheese, using diethyl ethoxymethylenemalonate as a derivatising agent. Food Chemistry 139(1-4):10291035. https://doi.org/10.1016/j.foodchem.2013.01.071

Rezac, S., C. R. Kok, M. Heermann, and R. Hutkins. 2018. Fermented foods as a dietary source of live organisms. Frontiers in Microbiology 9:1785. https://doi.org/10.3389/fmicb.2018.01785

Rhee, S. H., C. Pothoulakis, and E. A. Mayer. 2009. Principles and clinical implications of the braingut-enteric microbiota axis. Nature Reviews. Gastroenterology \& Hepatology 6(5):306-314. https://doi.org/10.1038/nrgastro.2009.35

Roberts, J. D., C. A. Suckling, G. Y. Peedle, J. A. Murphy, T. G. Dawkins, and M. G. Roberts. 2016. An exploratory investigation of endotoxin levels in novice long distance triathletes, and the effects of a multi-strain probiotic/prebiotic, antioxidant intervention. Nutrients 8(11):733. https://doi.org/10.3390/nu8110733

Robledo de Dios, T., M. Á. Dal Re Saavedra, C. Villar Villalba, and N. Pérez-Farinós. 2015. Contenido de ácidos grasos trans en los alimentos en España [Content of trans fatty acids in food products in Spain] Nutrición Hospitalaria 32(3):1329-1333. https://doi.org/10.3305/nh.2015.32.3.9410

Rossen, N. G., S. Fuentes, M. J. van der Spek, J. G. Tijssen, J. H. Hartman, A. Duflou, and C. Y. Ponsioen. 2015. Findings from a randomized controlled trial of fecal transplantation for patients $\begin{array}{llll}\text { with ulcerative } \quad \text { colitis. } & \text { Gastroenterology (1):110-118. }\end{array}$ https://doi.org/10.1053/j.gastro.2015.03.045

Schrezenmeir, J. and M. de Vrese. 2001. Probiotics, prebiotics, and synbiotics - approaching a definition. The American Journal of Clinical Nutrition 73(2):361s-364s. https://doi.org/10.1093/ajcn/73.2.361s

Smith, P. M., M. R. Howitt, N. Panikov, M. Michaud, C. A. Gallini, M. Bohlooly-Y, and W. S. Garrett. 2013. The microbial metabolites, short-chain fatty acids, regulate colonic Treg cell homeostasis. Science (American Association for the Advancement of Science, Washington, District of Columbia, USA) 341(6145):569-573. https://doi.org/10.1126/science.1241165

Steinkraus, K. 1992. 5. Lactic Acid Fermentations. In, Applications of Biotechnology to Fermented Foods: Report of an Ad Hoc Panel of the Board on Science and Technology for International Development. National Research Council (US) Panel on the Applications of Biotechnology to Traditional Fermented Foods. National Academies Press. Washington, District of Columbia, USA. 1992. https://www.ncbi.nlm.nih.gov/books/NBK234703/

Turnbaugh, P. J., F. Bäckhed, L. Fulton, and J. I. Gordon. 2008. Diet-induced obesity is linked to marked but reversible alterations in the mouse distal gut microbiome. Cell Host \& Microbe 3(4):213-223. https://doi.org/10.1016/j.chom.2008.02.015

van Zanten, G., A. Knudsen, H. Röytiö, S. Forssten, M. Lawther, A. Blennow, S. J. Lahtinen, M. Jakobsen, B. Svensson, and L. Jespersen. 2012. The effect of selected synbiotics on microbial composition and short-chain fatty acid production in a model system of the human colon. PLoS One 7(10)e47212. https://doi.org/10.1371/journal.pone.0047212

Verbeke, K., Boobis, A. Chiodini, A., Edwards, C., Franck, A., Kleerebesem, M., Nauta, A. Raes, J., van Tol, E., and Tuohy, K. 2015. Towards microbial fermentation metabolites as markers for health benefits of prebiotics. Nutrition Research Reviews 28:42-66. https://doi.org/ $10.1017 / \mathrm{S} 0954422415000037$

Wen, L. and A. Duffy. 2017. Factors influencing the gut microbiota, inflammation, and type 2 diabetes. The Journal of Nutrition 147(7):1468S-1475S. https://doi.org/10.3945/jn.116.240754 
Wilkin, D. and K. Akre. 2019. Lactic Acid Fermentation - Advanced. CK-12. https://www.ck12.org/biology/alcoholic-fermentation/lesson/Alcoholic-FermentationAdvanced-BIO-ADV/

Wilkin, D. and K. Akre. 2019. Alcohol Fermentation - Advanced. CK-12. https://www.ck12.org/biology/lactic-acid-fermenation/lesson/Lactic-Acid-FermentationAdvanced-BIO-ADV/

Williams, S. C. 2014. Gnotobiotics. Proceedings of the National Academy of Sciences of the United States of America 111(5):1661. https://doi.org/10.1073/pnas.1324049111

Woof, J. M. and M. A. Kerr. 2006. The function of immunoglobulin A in immunity. The Journal of Pathology 208(2):270-282. https://doi.org/10.1002/path.1877

Yang, J. Y. and M. N. Kweon. 2016. The gut microbiota: a key regulator of metabolic diseases. $B M B$ Reports 49(10):536-541. https://doi.org/10.5483/bmbrep.2016.49.10.144

Zhu, J. and W. E. Paul. 2008. CD4 T cells: fates, functions, and faults. Blood 112(5):1557-1569. https://doi.org/10.1182/blood-2008-05-078154 